Proc. Indian Acad. Sci. (Earth Planet. Sci.), Vol. 95, No. 3, November 1986, pp. 373-380.

(C) Printed in India.

\title{
An interpretation of induced magnetic variations near Shillong and Gulmarg
}

\author{
A K AGARWAL and H B NAIK \\ Indian Institute of Geomagnetism, Colaba, Bombay 400005 , India
}

MS received 14 November 1985; revised 30 July 1986

\begin{abstract}
Short-period events such as bays and storm sudden commencements (SSCs) have been analysed to investigate the nature of induced magnetic variations at two Indian magnetic observatories: Shillong and Gulmarg. It seems that near Gulmarg there is obvious connection between the induced magnetic variations and the two large scale features; the main central thrust (MCT) and the main boundary fault (MBF) in the northwest direction. The Dauki fault, an approximately east-west conductor, seems to be responsible for the conductivity anomalies at SHL.
\end{abstract}

Keywords. Induction; magnetic variations; horizontal disturbance vector.

\section{Introduction}

Gulmarg (GUL) and Shillong (SHL) are two permanent magnetic observatories situated in the Sub-Himalayan ranges of northern India (Lat: $34.01^{\circ} \mathrm{N}$, Long. $74.40^{\circ} \mathrm{E}$ for GUL and Lat. $25.57^{\circ} \mathrm{N}$, Long. $91.88^{\circ} \mathrm{E}$ for SHL). Their locations are shown on the map of India in figure 1a. Many of the deep-seated sub-surface geological features of their neighbouring regions (shown in figure 1b) can be studied through the anomalies in transient geomagnetic variations recorded at these two observatories. At SHL, earlier investigation by Agarwal et al (1979) through the analysis of short-time variations showed that $Z$ variations are largely suppressed. They attributed the cause to an eastward induced currents flowing close to the station. Recently Nityananda and Jayakumar (1981) also tried to give a geological interpretation on the basis of induction vectors. However, the path of induced currents could not be delineated. It is important to further investigate the induction in this region through the use of anomalies in horizontal components (H \& D) and to infer therefrom the exact nature of the induced current pattern in this geologically complex zone. At GUL, there have been no prior studies of induced magnetic variations. A simultaneous detailed investigation of these two stations (SHL \& GUL) can provide not only useful information about the likely behaviour of induced currents, but can also contribute to the fundamental understanding of the Himalayan geology.

To identify the character of sub-surface geological features in the vicinity of SHL \& GUL, we have analyzed the short-period events, such as bays and SSCs and compute through them the induction vectors and horizontal disturbance vectors. These parameters are very effective in identifying and locating internal currents causing the anomaly. Results of such analysis and their possible interpretation in terms of known geological features are reported here. 


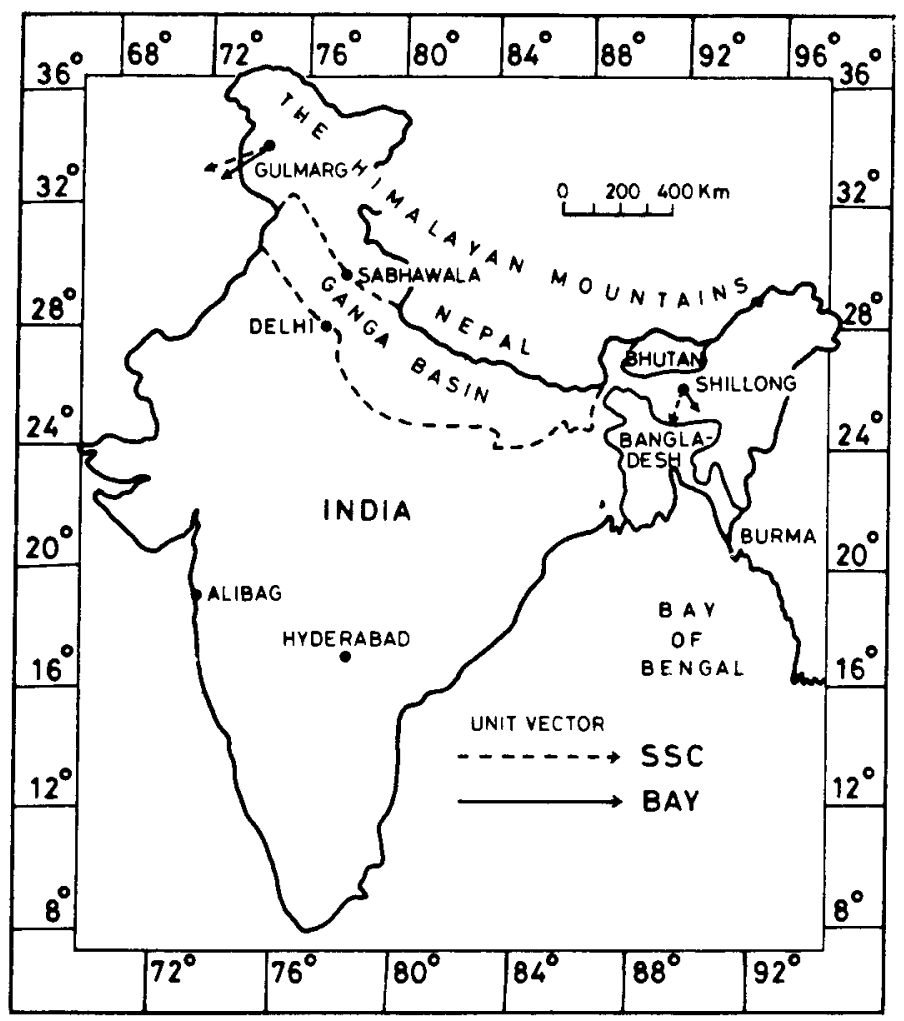

Figure 1 a. The induction vectors at Shillong and Gulmarg for bays and SSCs events.

\section{Data and analysis}

The magnetic variations during a typical night-time bay at the northern stations, GUL \& SHL, are shown in figure 2. In the figure the magnetic variations at Alibag (ABG) are also included for comparison. Their features exhibit the following important characteristics in comparison to $\mathrm{ABG}$ (i) Both $Z$ \& $D$ variations are suppressed at SHL, (ii) $Z$ variations at SHL \& GUL are positive in contrast to negative $Z$ variations at $A B G$ which happens to be a coastal station and (iii) Enhanced $H$ variations at GUL compared with that at ABG \& SHL. This enhancement is more pronounced for short-period night-time events such as SSCs. The latitudinal dependence of amplitude of $D$-variations from ABG to GUL does not fit into the trend of uniform amplitude variations of the horizontal components (H \& D) over these latitudes (Schmucker 1969).

In order to investigate the nature of induction vectors, about 30 night-time bays and SSCs have been scaled from magnetograms to give amplitudes of $\Delta H, \Delta D, \Delta Z$. The changes in amplitudes were measured by taking the basevalue as the value at the start of the event. The observed set of $\Delta Z$ is fitted to corresponding $\Delta H$ and $\Delta D$ 


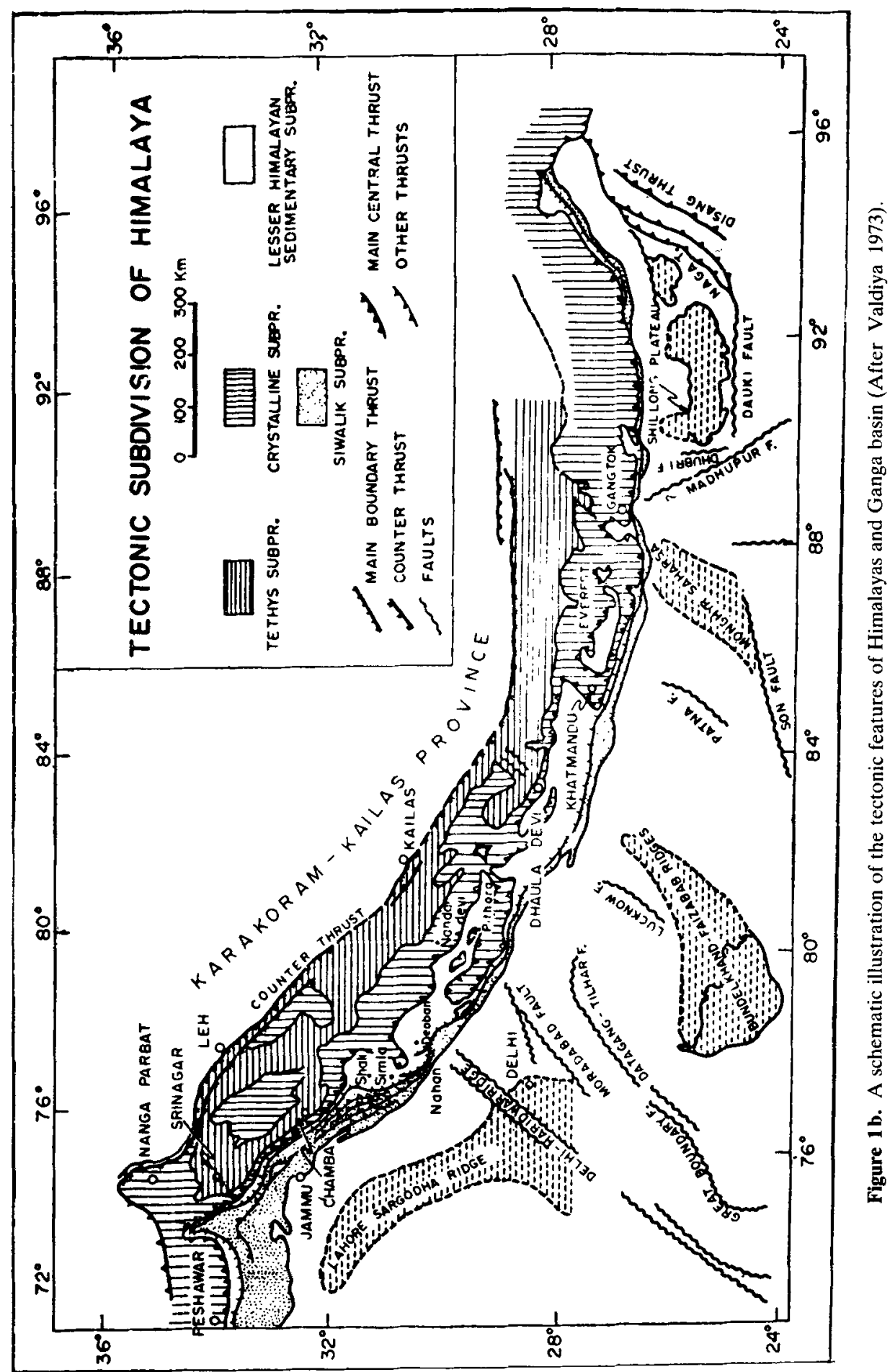




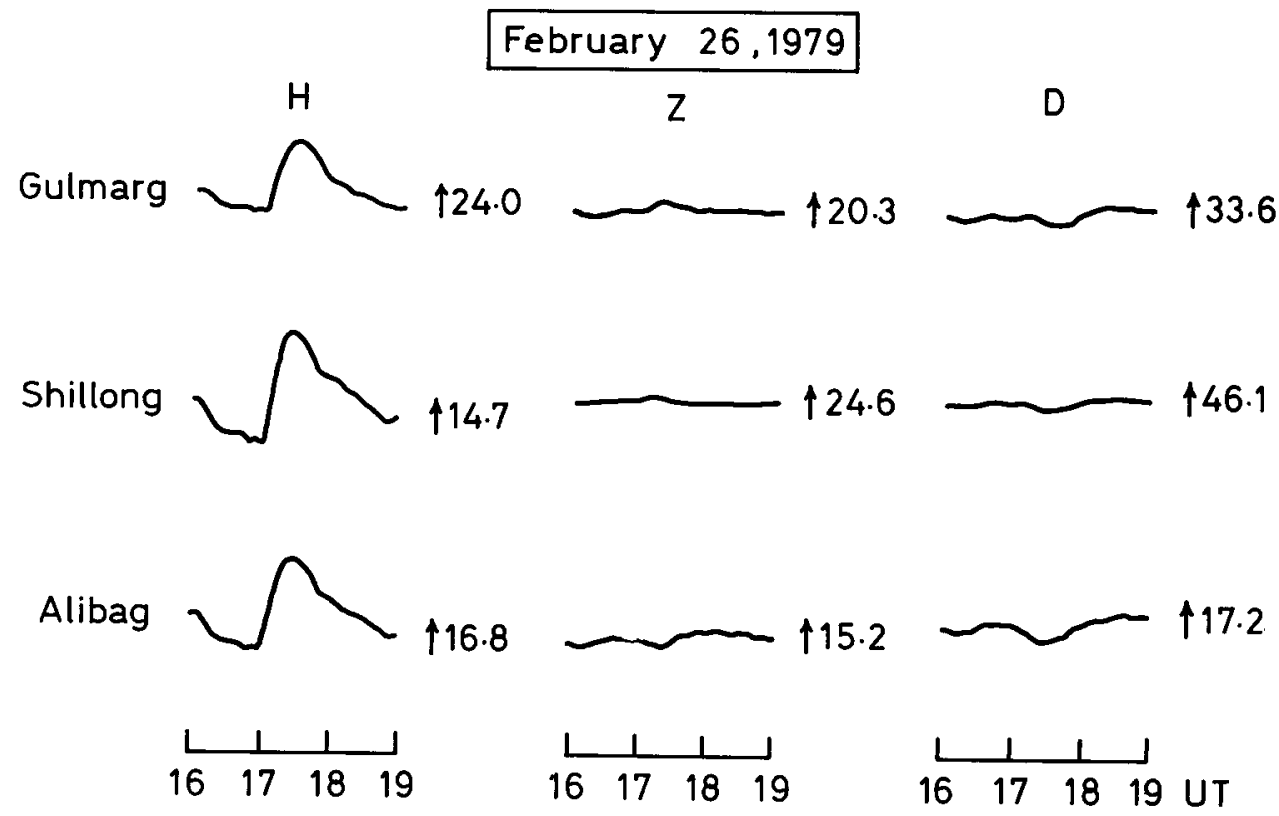

Figure 2. Amplitude variations during a typical magnetic bay observed at low-latitude Indian stations. Units marked are scale values, $n \mathrm{~T} / \mathrm{cm}$. Time marked on $X$-axis is in UT.

Table 1. The magnitude $(\vec{S})$ and direction $(\theta)$ of induction vectors obtained at Shillong and Gulmarg for both bays and SSCs

\begin{tabular}{|c|c|c|c|c|c|}
\hline \multirow[b]{3}{*}{ Station } & \multirow[b]{3}{*}{ Code } & \multicolumn{4}{|c|}{ Induction Vector } \\
\hline & & \multicolumn{2}{|c|}{ Bays } & \multicolumn{2}{|c|}{ SSCs } \\
\hline & & $S$ & $\theta^{*}$ & $S$ & $\theta^{*}$ \\
\hline Shillong & SHL & $0 \cdot 08$ & $336 \cdot 0^{\circ}$ & $0 \cdot 22$ & $19 \cdot 3^{\circ}$ \\
\hline Gulmarg & GUL & $0 \cdot 36$ & $65 \cdot 7^{\circ}$ & 0.42 & $78 \cdot 6^{\circ}$ \\
\hline
\end{tabular}

* measured clockwise from south

in a least square sense to estimate coefficients $A$ and $B$ by minimizing the effect of $\varepsilon$ in the following equation:

$$
\Delta Z=A . \Delta H+B . \Delta D+\varepsilon
$$

Here $\varepsilon$ denotes the uncorrelated part of $\Delta Z$. The coefficients were then combined to give an induction vector $\vec{S}\left[=\left(A^{2}+B^{2}\right)^{1 / 2}\right]$ and its azimuth $\theta=\tan ^{-1} B / A$. The induction vectors, so obtained, for bays and SSCs at SHL \& GUL are shown on the map of India (figure. 1a) and their values are given in table 1.

To determine whether an anomaly in the horizontal components exists or not, the latitudinal trend of mean directions of horizontal variations at GUL and SHL are compared with the mean direction at $\mathrm{ABG}$, with the assumption that horizontal variations at Alibag are normal (Nityananda et al 1977). For computing mean 
directions of horizontal variations at the three stations, the amplitude of selected events was plotted with $\Delta H$ (in nanoteslas) on the $Y$-axis (representing North) and $\Delta D$ (also in nanoteslas) on the $X$-axis (representing East). The least square fit of mean direction to scatter of points for all the three stations are shown in figure 3 for bays and SSCs separately. In both class of events, the mean directions at SHL \& GUL do not fit in the latitudinal trend of gradual shift towards east, indicating an anomalous behaviour at these stations. At SHL, the change in direction is slightly more eastward for SSCs whereas for bays it is west of ABG. GUL also shows similar changes as that of SHL. These changes in the mean directions indicate the presence of magnetic anomalies in horizontal components at both stations.

For identifying the nature of the anomalies in the horizontal components, we computed horizontal disturbance vectors $\left(\Delta \vec{B}_{A}\right)$ following the procedure given in Schmucker (1969). First, the anomalous part in the horizontal components $\left(\Delta H_{A}\right.$ and $\Delta D_{A}$ ) at GUL \& SHL were calculated by taking the algebraic difference between the horizontal components as recorded at these stations and the corresponding value recorded at $\mathrm{ABG}$ for all possible common events. The anomalous parts $\Delta H_{A}$ and $\Delta D_{A}$ are plotted again taking $\Delta H_{A}$ on the $Y$-axis and $\Delta D_{A}$ on the $X$-axis. Figure 4 shows the plots for both stations and for bays and SSCs. The mean direction of the vectors was obtained by the least square fit. The direction of induced currents can be obtained by rotating this vector anticlockwise through $90^{\circ}$ (Schmucker 1969).
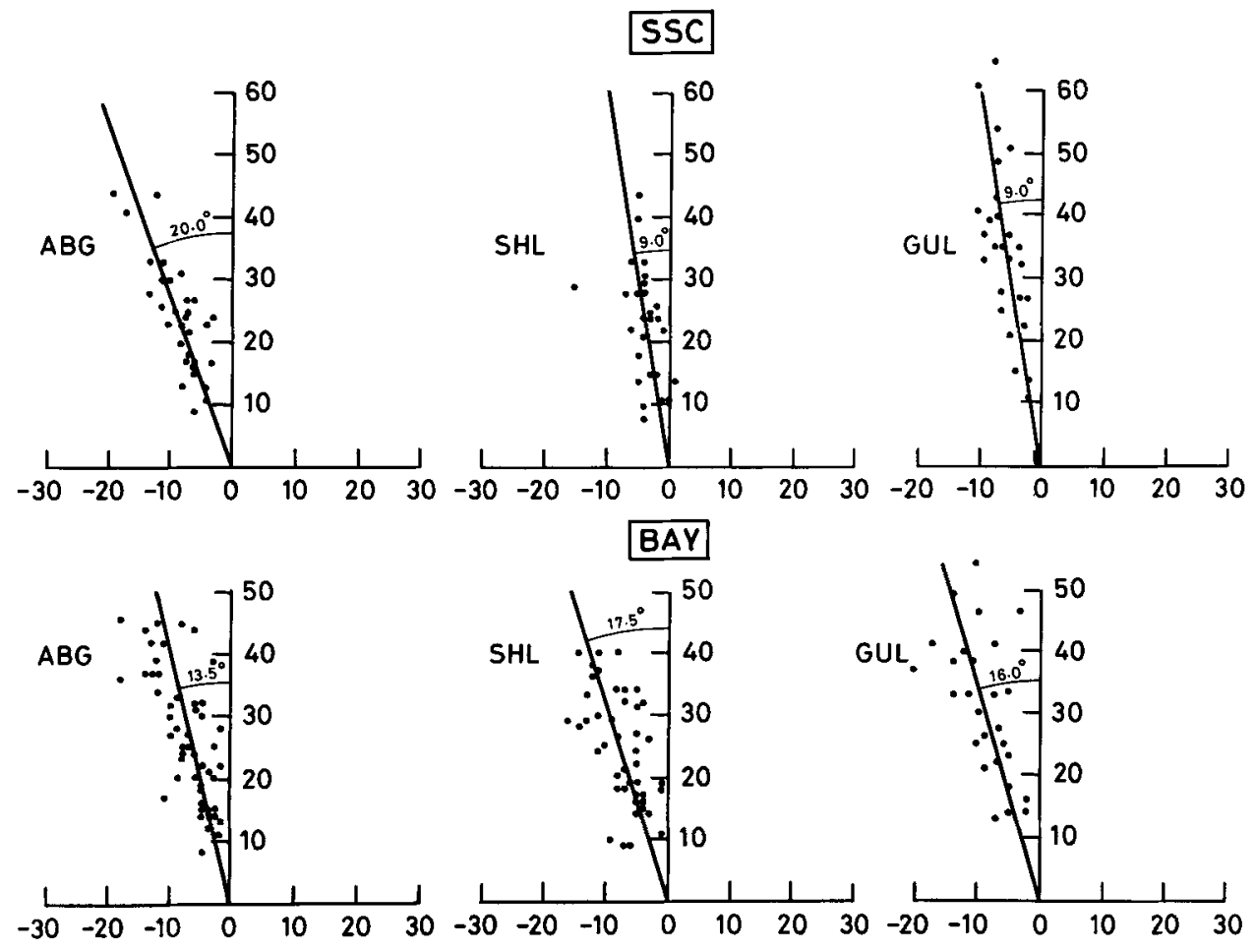

Figure 3. Mean direction of horizontal variations at Alibag, Shillong and Gulmarg for bays and SSCs events. 


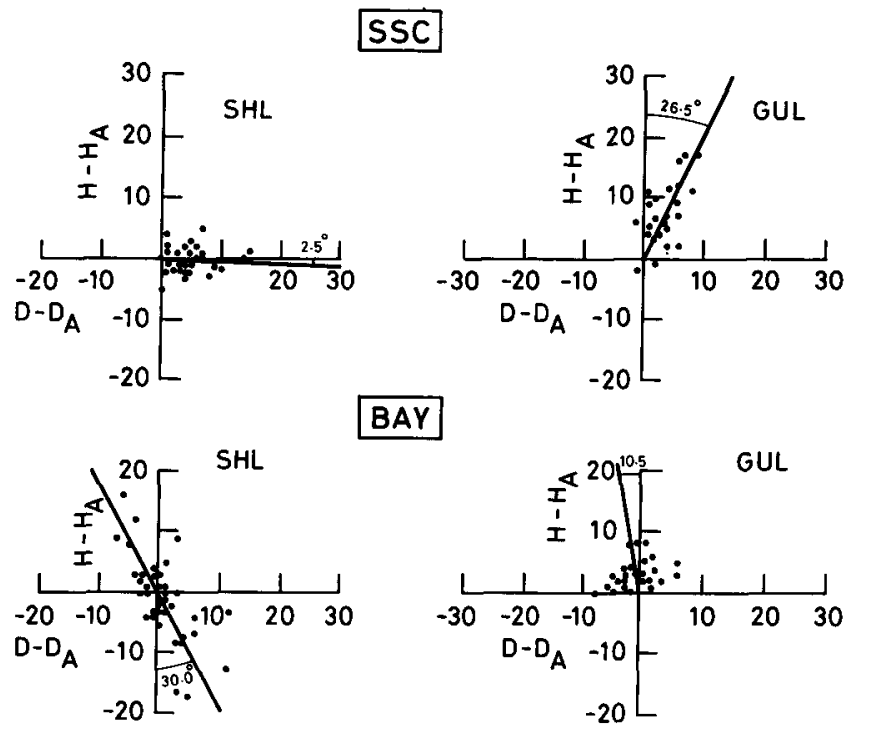

Figure 4. Mean direction of anomalous horizontal variations at Shillong and Gulmarg is obtained after subtraction of the corresponding amplitudes observed at Alibag $\left(H_{A}\right.$ and $D_{A}$ ) which is taken to be a normal station. Units marks on the axis are nT.

\section{Results and discussion}

It is seen from the nature of induction vectors (figure 1a) that the conductor causing the anomaly at GUL lies approximately south-west of that station, and it is towards south or south-east at SHL. The directions of induced electric currents inferred from the anomalous horizontal variations $\Delta \vec{B}_{A}$ are found to be $79 \cdot 5^{\circ}$ (measured clockwise from the south) for bays and $116.5^{\circ}$ for SSCs at GUL whereas it is about $240.0^{\circ}$ and $182.5^{\circ}$ for bays and SSCs respectively at SHL. It implies that induced current would flow north-west or westward near GUL. At SHL, the direction of induced electric currents inferred would be north-east or eastward for bays whereas for SSCs it would be northward.

The position of GUL in the Himalayan ranges makes an east-west conductor easy to conceive of. The trend of the Himalayan chain is approximately east-west, a rather wide arc swinging from north-west to south-east. All the structural features linked with the Orogeny will also have a dominant east-west trend. Of the large scale features, the two main thrusts: (i) the main central thrust and (ii) the main boundary fault run parallel to each other along the range and their main tectonic trend (figure $1 \mathrm{~b}$ ) is in conformity with the direction of induced currents. These structural features of the Himalayas (possibly an asthenosphere upwelling between the two faults) seem to be responsible for the conductivity anomalies at GUL. It is important to note that between these two faults the lesser Himalayan sedimentaries lie in tangled confusion of structure and stratigraphy (Valdiya 1964). On the south of the main boundary fault extends a vast group of Siwalik and older tertiary sediments whose thickness is about $5 \mathrm{~km}$. These layers of sediments will also affect the short-period fluctuations in the magnetic records of GUL. A schematic 
illustration of the tectonic features of Himalayas and Ganga basin is presented in figure $1 \mathrm{~b}$ after Valdiya (1973).

On the consequence of Himalayan Orogeny, step-like rise of the asthenosphere along the entire range would provide a sharp vertical lateral conductivity contrast. Such a step-like structure can also contribute to the induced magnetic variations at GUL. A similar type of structure has been proposed by us for the conductivity anomalies at Sabhawala (Nityananda et al 1981). Recently Srivastava (1981) also studied the night-time bays observed at Sabhawala, Yangi-Bazar (Tashkent) and Alma Ata observatories and proposed the cause of anomaly to be induced currents in a step-structure at the Moho boundary (upper mantle material under the plain juxtaposed against the crustal material beneath the Himalayas) in an east-west direction along the junction of Himalaya foothills. However, it is important to note that the effect of sedimentary basin in Indo-Gangetic plain is not very distinct as the induction vectors for both bays and SSCs point towards south-west instead of towards south or south-east.

To investigate the nature and depth of the conductor, we have utilized horizontal disturbance vector $\left(\Delta \vec{B}_{A}\right)$ [where $\left.\Delta B_{A}=\Delta H_{A}^{2}+\Delta D_{A}^{2}\right)^{1 / 2}$, we have computed the modulus of ratio $\Delta B_{A} / \Delta Z_{A}$ for each event and then averaged them over all the events in each class. The anomalous variation $\left(\Delta Z_{A}\right)$ is taken to be the total amplitude of the observed variation in the vertical component. Lilley (1976) has suggested that a response arrow scaled as arctan $\Delta B_{A} / \Delta Z_{A}$ be plotted in the

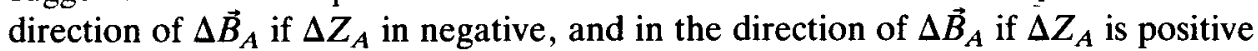
thereby obtaining an arrow whose length is maximal and finite directly over the current. The magnitude of these response arrows together with standard deviations is found to be $1.33 \pm 0.66$ for SSCs and $0.93 \pm 0.37$ for bays at GUL. The nature of the response arrows indicates that, for periodicities around SSCs, induced currents are close to it and they flow at a shallow depth. At longer periods (i.e. for bays), the currents not only flow at shallower depth (sedimentary layer) but also in the upper mantle thereby smearing the overall induced effects at such periods. This is indicated by the smaller value of the response arrow for the bays.

At SHL, the location of induced currents is very close to the station but slightly towards south is provided by the negligible positive $Z$-variations (figure 2 ) and the nature of inductor vectors (figure 1a). The magnitude of the response arrows computed for bays $(1.23 \pm 0.49)$ and SSCs $(0.94 \pm 0.31)$ suggest that the conductivity contrast existing at much deeper level is much closer to the station than that of the shallow feature. On the basis of known geological features existing here, we find that the prominent Dauki fault (figure 1b) striking E-W, lying south of the Shillong plateau is a deep-seated fault and seems to be responsible for the induced geomagnetic variations at SHL as suggested by Nityananda and Jayakumar (1981). There is also a possibility of contribution from a conducting path in the rise of mantle south of Shillong Plateau. This is because the crustal thinning is much more steep toward Surma Valley than that of Brahmaputra valley (Verma and Gupta 1973). Another important factor contributing to the induced variations at SHL seems to be due to large sedimentary deposition in the Surma Valley in the northward direction (in conformity with the induced current direction for SSCs). In this valley the thickness of sediments is known to be about 10-12 km (Verma and Gupta 1973). This layer will affect the short-period variations in a more pronounced way. Thus this station seems to be affected by two conductors: one 
approximately in the north-east or eastward direction (deep seated structure) and another in a northward direction (shallow structure). Since the contribution of induced effects to horizontal variations has been estimated by taking Alibag (ABG) as a normal station which is sufficiently far away in longitude from this station, further confirmation of the results is necessary when some more data from a network of temporary stations are available in this geologically complex zone. The large value of scatter in the magnitude of the response arrows at this station is attributed to the small $Z$-variations and to the assumption that total $\Delta Z$ observed is wholly anomalous.

\section{Conclusions}

The direction of induced currents inferred from the nature of the horizontal anomalies $\left(\Delta \vec{B}_{A}\right)$ indicate that the conductivity contrast, possibly an asthenosphere upwelling between two faults: main boundary fault and the main central thrust, is the cause of conductivity anomalies at GUL. South of the main central thrust, the lesser Himalayan sediments and the Siwalik sediments also affect the short-period fluctuations. At SHL, an approximately east-west conductor, namely Dauki fault, together with dense sediments in the Surma valley seems to be responsible for the conductivity anomalies. The induced effects are more pronounced for shorter periods (i.e. SSCs) than for longer periods (i.e. bays).

It is interesting to note that induced currents in the Himalayan region tend to flow parallel to the main tectonic trend which is more or less NW-SE near GUL and NE-SW in Assam near SHL.

\section{Acknowledgement}

The authors are grateful to Prof. R G Rastogi and Prof. B P Singh for many useful discussions and their encouragement during the progress of this work.

\section{References}

Agarwal A K, Singh B P and Nityananda N 1979 Phys. Earth Planet. Inter. 18232

Lilley F E M 1976 Geophys. J. R. Astron Soc. 46165

Nityananda N and Jayakumar D 1981 Proc. Indian Acad. Sci. (Earth Planet. Sci.) 9047

Nityananda N, Agarwal A K and Singh B P 1977 Phys. Earth Planet. Inter. 155

Nityananda N, Agarwal A K and Singh B P 1981 Phys. Earth Planet. Inter. 25226

Schmucker U 1969 in Applications of modern physics to the earth and planetary interiors (ed) S K Runcom (New York: Interscience) 125

Srivastava B J 1981 Phys. Earth Planet. Inter. 25210

Valdiya K S 1964 Rep. 22nd Int. Geol. Congr. 1115

Valdiya K S 1973 Geophys. Res. Bull. 1179

Verma R K and Gupta R P 1973 Tectonophysics 1819 